\title{
An improved method for accurate computation of coastal upwelling index using Sea Surface Temperature Images
}

\author{
Anass El Aouni ${ }^{1,2}$, Khalid Minaoui ${ }^{1}$, Ayoub Tamim ${ }^{1}$, Khalid Daoudi ${ }^{2}$, Hussein Yahia ${ }^{2}$ and Driss Aboutajdine ${ }^{1}$ \\ ${ }^{1}$ Mohammed V-Agdal University, LRIT Associated Unit to the CNRST-URAC n 29, Rabat, Morocco \\ ${ }^{2}$ INRIA Bordeaux Sud-Ouest (GEOSTAT team), Talence, France
}

\begin{abstract}
In this paper we aim to present a new methodology to derive rigorous SST-based coastal upwelling index for the purpose of conducting a saisonal variablity of upwelling area along the Moroccan Atlantic coast. The method is based on the scientific knowledge of upwelling area and its spatial distribution provided by expert oceanographers. The latter consists in automatically identify and extract the region covered by the upwelling waters in the costal ocean of Morocco using the Fuzzy c-means algorithm and finding regions of homogeneous pixels. Then Region Growing process is used to filter out the remaining noisy structures in the offshort waters. The methodology is used to provide a satistical view of the spatial and temporal variability of the Moroccan upwelling activity. The relevance of the proposed Coastal Upwelling Index (CUI) is evaluated by an oceanographer using 86 8-days sea surface temperature images and it is shown to be superior to that of the standard upwelling index.
\end{abstract}

Index Terms-Sea surface temperature, Coastal Upwelling Index, Fuzzy clustering algorithm, unsupervised classification.

\section{INTRODUCTION}

$\mathbf{T}$ HE coastal ocean of Morocco which is part of the Eastern Boundary Upwelling Ecosystems is characterized by the presence of upwelling phenomenon. This coast is forced by the equator-ward trade winds that lead to the upward pumping of cold intermediate water. The phenomenon can be identified by seasonally variable low sea surface temperature (SST) and nutrient-rich water in coastal area.

The Moroccan coastal upwelling is a part of The canary current system that extends from the Iberian Peninsula to the south of Senegal, characterized by persistent and variable upwelling all around the year, making it rich in fishery resources [7]. In fact, the evolution of the pelagic ecosystem of this region is influenced by the high dynamics and variability in space and time of this phenomenon largely dependent on the winds regime changes.

The classical way to quantify the intensity of upwelling is by computing Ekman transport. The wind-based upwelling index can be interpreted as the water flux theoretically transported offshore by the wind stress from the coastal upward flux of colder water, assuming an infinite ocean [6]. But this classical way do not consider bathymetricy the continental shelf and therefore cannot render the complexity of the two dimensional spatial structure of the upwelling such as coastal upwelling cells, cape effects and filaments [2].
For this purpose, the remotely Sea Surface Temperature (SST) provides a quantitative and synoptic overview of thermal characteristics in the ocean including the surface of upwelled water which is a potential proxy of the upwelling intensity. In fact, the remote sensing is widely applied technique in the detection of coastal upwelling [1]. particularly, the thermal infrared image of the ocean obtained from the Moderate Resolution Imaging Spectroradiometer (MODIS) sensor aboard Terra spacecraft, are frequently used to detect the thermal upwelling front. Generally, the detection of upwelling areas has been made by oceanographers using high resolution color scale map for each image, which can be very time consumingprocess due to the enormous amount of daily data.

The remainder of this work is structured as follows: Section II presents the database used throughout this work. Section III and IV describes methodologies used to improve the SSTbased coastal upwelling index, while section $\mathrm{V}$ reports the experimental results and analysis. Finally, a conclusion is drawn in section VI.

\section{REGION OF INTERSET AND DATASETS}

\section{A. MODIST SST}

MODIS-T SST data were extracted from the Physical Oceanography Distributed Active Archive Center (PODAAC) with spatial resolution of $4 \times 4 \mathrm{~km}$, covering the southern part of the Moroccan Atlantic coast spans from $20^{\circ} 380$ $28^{\circ} 270 \mathrm{~N}$ and $11^{\circ} 170-23^{\circ} 00 \mathrm{~W}$. Each SST image is defined by $176 \times 176$ pixels, and each pixel contain temperature information in Celsius degrees.

8-day MODIS-T SST images are used to provide a coarse segmentation of upwelling areas, covering the years 2007 and 2008. the weekly synthetic data is chosen as a compromise between the variability of the pixel values and the necessity to provide a continuous spatio-temporal coverage. A color scale is applied to our database, in order to help the oceanographer, getting the best contrast definition for a good visualization of upwelling area. Fig. 1 shows an image selected from our database illustrating an upwelling scenario encountered throughout this work. 


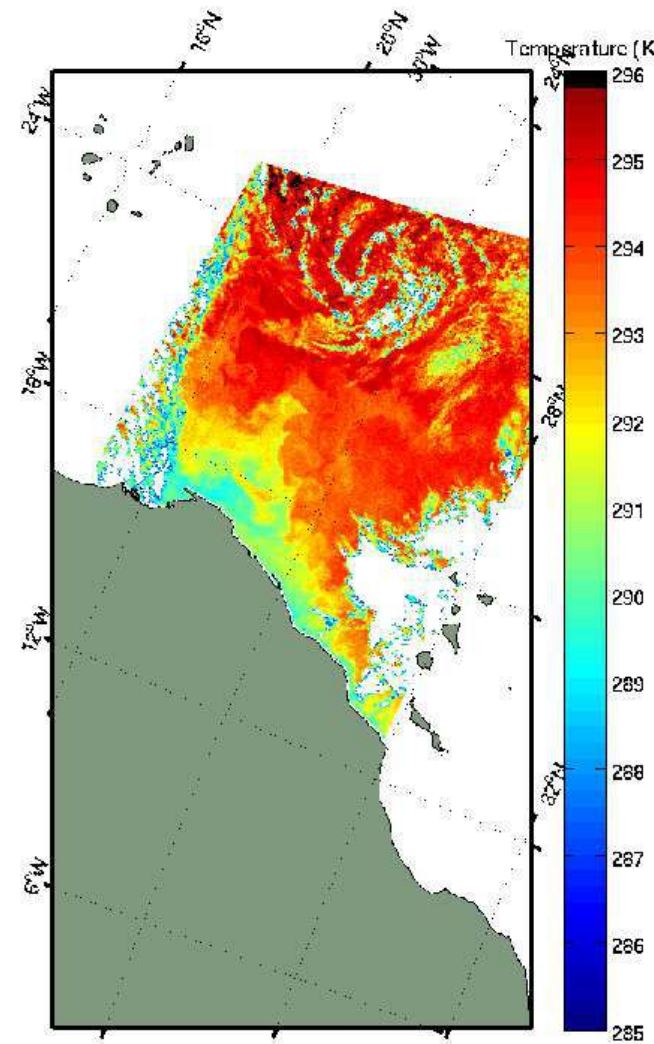

Fig. 1: SST image obtained on 2007-10-29.

\section{UPWELLING DETECTION METHODOLOGY}

\section{A. Clustering technique}

In clustering [14] we try to identify groups of pixels because they are somehow similar to each other, when the only data available are unlabeled, and no structural information about it is available. Depending on the nature of the data and the purpose for which clustering is being used, different measures of similarity may be used to place items into classes.

The existing clustering methods can be grouped into two categories, fuzzy [11] and hard [9]. In hard clustering, data is divided into distinct clusters, where each data element belongs to exactly one cluster. In fuzzy clustering, data elements can belong to more than one cluster simultaneously, with different degrees of membership.

One of the most widely used fuzzy clustering algorithms is the fuzzy c-means algorithm, which attempts to partition a finite collection of $n$ pixels $X=x_{1}, \ldots, x_{n}$ into a collection of $c$ fuzzy clusters with respect to some given criterion. Given a finite set of pixels, the algorithm returns a list of $c$ cluster centres $C=c_{1}, \ldots, c_{c}$ and a fuzzy partition matrix, which presents the degree of belonging for each pixel $x_{k}$ to a given cluster $c_{i}$. The FCM algorithm is applied to minimize an objective function called c-means functional:

$$
J_{m}(X ; U, V)=\Sigma_{k=1}^{n} \Sigma_{i=1}^{c}\left(\mu_{i k}\right)^{m}\left\|x_{k}-v_{i}\right\|^{2}
$$

where $n$ is the length of the vector $X$ presenting pixels of a given SST image. $V=\left\{v_{1}, v_{2}, \ldots ., v_{n}\right\}$ vector of cluster prototypes and $U=\left[\mu_{i k}\right]$ is the fuzzy partition matrix which presents the degree of belonging for each pixel $x_{k}$ to a given cluster $c_{i}$. The weighting exponent $m$ controls the fuzziness of membership values, which vary from $[1, \infty[$. An increase of $m$ lead to an increase of fuzziness partitions. In our work, we set this parameter to 2 .

The objective function (1) can be seen as a measure of the total variance of $x_{k}$ from $v_{i}$. The minimization of the objective function (1) represents a nonlinear optimization problem that can be solved by using respectively the following cluster centroids and membership functions :

$$
\begin{gathered}
v_{i}=\frac{\sum_{k=1}^{n}\left(\mu_{i k}^{m}\right) x_{k}}{\sum_{k=1}^{n}\left(\mu_{i k}^{m}\right)}, 1 \leq i \leq c \\
\mu_{i k}=\frac{1}{\sum_{j=1}^{c}\left(\frac{\left\|x_{k}-v_{i}\right\|}{\left\|x_{k}-v_{j}\right\|}\right)^{\frac{2}{(m-1)}}}, 1 \leq i \leq c, 1 \leq k \leq n
\end{gathered}
$$

The goal here is to iteratively improve a sequence of sets of fuzzy clusters through those equations until no further improvement is possible for the objective function.

\section{B. Optimal number of clusters}

In unsupervised classification, the number of clusters is rarely known apriori, and in order to have the optimal number of classes it must be chosen with great care. Several methods have been proposed to automatically determinate the number of clusters which better fits the data [8], which can be done by using validity indices for several values of $c$ and then evaluate the goodness of the obtained c-partition, which can be very time consuming for large datasets. Also depending on the nature of the datasets that present spatial information, which can be the main limitations of these indices.

Due to the nature of our study area, covering the southern part of Moroccan Atlantic coast, characterized by a strong and variable upwelling throughout the year, and based on the scientific and technical knowledge of the coastal ocean of Morocco, we thus set the number of clusters to $2(c=2)$ in order to separate the cold water near the coast form the warmer in the offshort direction.

Fig. 2 shows the image result after the upwelling detection methodology. The red region on the image corresponds to upwelling area, and the blue pixels correspond to offshore waters non affected by the coastal upwelling. Whereas the white color correspond to the missed data.

\section{Upwelling extraction}

In addition to the Upwelling area, The Upwellig detection's output include some additional noise structures faraway from the coast, not belonging to the upwelling region such as cloud not properly masked and isolated cold water. For that reason and based on the fact that all pixels pertaining to the upwelling must have connectivity with the coastline, a segmentation procedure is required consisting on the application of the 


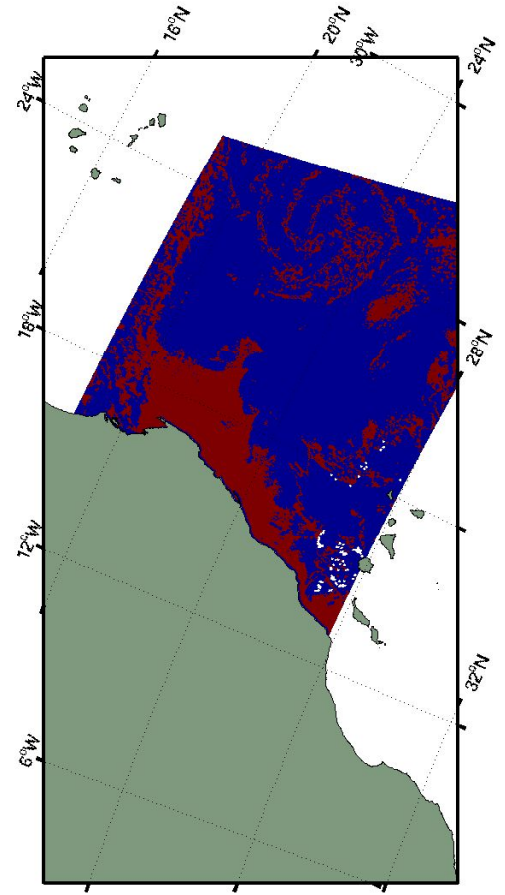

Fig. 2: Binary image result of the SST image in the Fig. 1, after the application of the FCM algorithm.

Region-growing algorithm to remove those isolated pixels in the offshore direction.

The Region-growing algorithm is a simple region-based image segmentation method that examines neighboring pixels of initial seed points and determines whether the adjacent pixel should be added to the region [3][16]. This method aims at grouping a set of pixels according to homogeneity and adjacency criteria [13]. In this work, we have chosed 8connected neighborhood for the adjacency criteria. Since the output of the upwelling detection step present a binary image, the stop condition is the value 0. Fig. 3 shows the results of the Region-growing algorithm applied to the binary images of Fig. 2, where remaining pixels in offshore waters have been removed. Fig, 4 shows the final segmentation achieved by the proposed methodology, where the upwelling areas are automatically contoured.

The proposed methodology is used to improve the SSTbased coastal upwelling index based on the nature of the upwelling area which can be defined by a continuous area of minimum SST, and can be done by using FCM clustering algorithm.

The way both thermal references were chosen in the literature was always arbitrary. Our contribution to this classical SST-based upwelling index is to improve the way to properly define and extract these two thermal references.

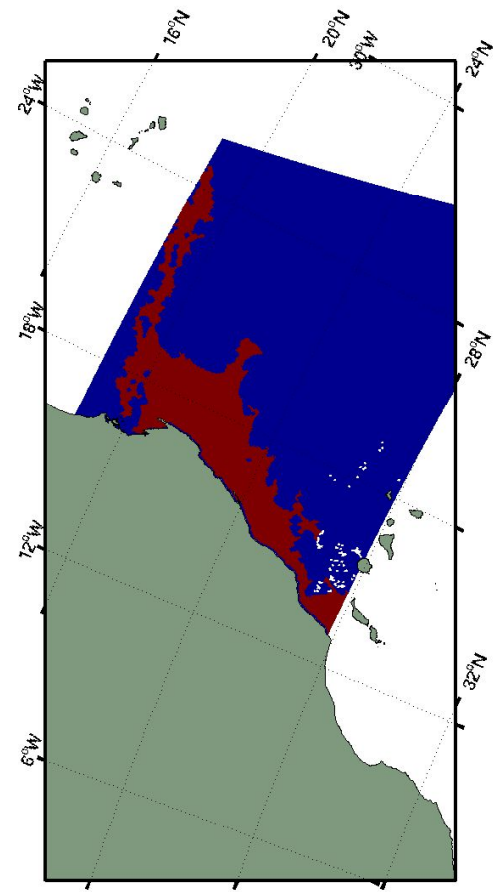

Fig. 3: Binary image result of the SST image in the Fig. 1, after the application of the Region-growing algorithm.

\section{SST-BASED UPWELLING INDEX}

Many methods have been proposed in the literature [17] [5] as a simple thermal difference $(\Delta T)$ between the coastal and the offshore SST taken at the same latitude, in order to estimate the latitudinal and temporal variability of the intensity of the upwelling. Formulation is therefore very simple:

$$
C U I_{s s t}=S S T_{\text {offshore }}(\max )-S S T_{\text {inshore }}(\min )
$$

\section{A. Identification of the $S S T_{\min }$}

All previous $S S T_{\min }$ are defined as the minimum $S S T$ recorded in the coastal band from the coast up to the distance of the continental slope. Whereas the coastal reference itself is measured over various widths. The most coastal $S S T$ chooses $500 \mathrm{~km}$ from the coast as the $S S T_{\min }$ reference. Whereas [12] used it as the offshore position.

In our work the $S S T_{\min }$ is defined as the minimum value measured along the perpendicular line to the parallel of the coastline within the extracted upwelling area with FCM clustering algorithm, to avoid having a static upwelling area. Let $G_{c}\left(p_{c}(i, j) \ldots p_{c}(i, j)\right)$ be group of pixel with Homogeneous temperature, where $c$ is the number which represent the Upwelling cluster. and $f_{1}(x, y) \ldots f_{k}(x, y)$ perpendicular lines to the coast.

the vector of the minimum SST values is represented as: 


$$
S S T_{\min 1 \ldots k}=p\left(x_{i}, y_{i}\right),\left\{\begin{array}{c}
\underset{x i, y i}{\arg \min } p(x, y) \\
\left(x_{i}, y_{i}\right) \epsilon f_{1 \ldots k}(x, y) \\
p\left(x_{i}, y_{j}\right) \epsilon G_{c}
\end{array}\right.
$$

\section{B. Identification of the $S S T_{\max }$}

The maximum temperature, noted $S S T_{\max }$ is chosen as the offshore temperature, which used to be the coastal SST where the influence of the upwelling is expected to be insignificant. All preceding SST-based indices make chose $S S T_{\max }$ from various locations at arbitrary distances from the coast, [12] choose the offshore position at $500 \mathrm{~km}$ from the coast,[15] found that the general patterns of the spatio temporal variability of their SST-based index were similar within the range 400-1000 km offshore. [10] chose the SST average in the band located within $500-700 \mathrm{~km}$ from the coast.

In our work we use a fixed distance of $700 \mathrm{~km}$ outside the extracted upwelling area, which make it a dynamic area to the coast line in latitude according the intensity of the upwelling, following the same perpendicular line to the parallel of the coastline used before to calculate the $S S T_{\min }$. The vector of the maximum SST values is represented as:

$$
S S T_{\max } 1 \ldots k=p\left(x_{i}, y_{i}\right),\left\{\begin{array}{c}
\underset{x i, y i}{\arg \max } p(x, y) \\
\left(x_{i}, y_{i}\right) \in f_{1 \ldots k}(x, y) \\
p\left(x_{i}, y_{j}\right) \epsilon G_{o f f}
\end{array}\right.
$$

where $G_{o f f}$ is the group of Homogeneous pixel that represent the offshore waters. Fig. 4 shows how both $S S T_{\min }$ and $S S T_{\max }$ must be chosen.

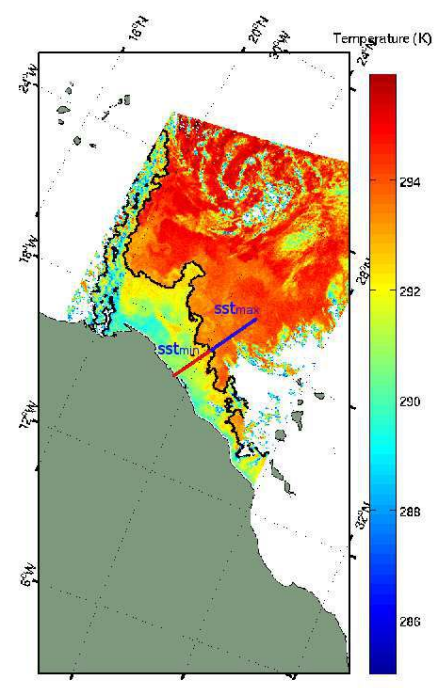

Fig. 4: Final segmentation achieved by the proposed methodology where the upwelling was delimited by the black color. Example of how both $s s t_{\min }$ and $s s t_{\max }$ were chosen within a perpendicular radial to the coast

\section{EXPERIMENTAL RESULTS AND ANALYSIS}

Based on well-known fact that validation in the case of ocean data is often and truly performed by assessing the results by professional oceanographers [4], the performance of the proposed methodology has been validated by the oceanographer over the data set of 86 SST images, basing on the scientific and technical knowledge of the Moroccan atlantic coast.

Throughout this evaluation, we used 4 grades: "Poor", "Acceptable", "Good" and "excellent" to check if the proposed yield to a satisfactory identification of the upwelling area without over or missing-detection. "poor" is assigned when the upwelling areas is not well delimited by the algorithm, and "excellent" was assigned when the areas is well delimited.

The results of the oceanographer interpretation for the proposed methodology are presented in Fig. 8, as we can see the grade "poor" reached the value $4.6 \%$ demonstrating the robustness of the proposed methodology in term of over or missing-detection. For the "Good" and "Excellent" values of $50 \%$ and $38.4 \%$ are achieved. Overall, $88.4 \%$ were reached by the two grades "Good" and "Excellent" together.

After evaluating the proposed methodology, over this representative database, we can conclude that the proposed algorithm has provided satisfactory and promessing results.

The calssical CUI used to choose the $S S T_{\min }$ within a random static area, while our one use to extract it from an homogeneous and continuous area of minimum SST extracted using the FCM algorithm. The proposed method avoid falling in spatial isolated pixel, not belonging to coastal upwelling such isolated cold waters and cloud pixels, as shown in Fig. 5 where the only homogeneous pixels representing the upwelling area were contoured. While using the classical way, the $S S T_{\min }$ will falls on one of those pixels, giving by that a wrong spatial upwelling intensity as shown in Fig. 6. In this figure, the intensity is very higher between latitudes $26^{\circ} \mathrm{N}$ - $28^{\circ} \mathrm{N}$ and $25^{\circ} \mathrm{N}-25.5^{\circ} \mathrm{N}$ due to the presence of cloud pixels and isolated cold water respectively. However a wrong spacial upwelling intensity can be easly detected, while it's impossible when it comes to the seasonal variability, which is computed by averging spatial intensities of the upwelling in a given period of time, including those miscalculated intensities, giving by that wrong seasonal variability of the $C U I_{s s t}$.

The upwelling area is presented as the homogeneous areas near the coast presenting the cold upward waters, which can take various forms and extensions due to the spatial and seasonal variability of the wind stress, and by using the classical way, we may skip some inshore minimum $S S T$ due the the arbitrary offshore position.

The proposed methodology is used to provide a spaceseasonal variability of the CUI, and this Coastal Upwelling Index is analyzed by averaging the latitudinal intensities of upwelling in a given season. Fig. 7 shows a spacial variability of the upwelling intensity all around the year, presenting the same tendency fo the 4 seasons in general, with important intensity in the summer, where the upwelling intensity is higher along the whole coast with maximal value of $6^{\circ}$ caught between latitude $22^{\circ} \mathrm{N}$ and $22.5^{\circ} \mathrm{N}$. In the winter season, the 


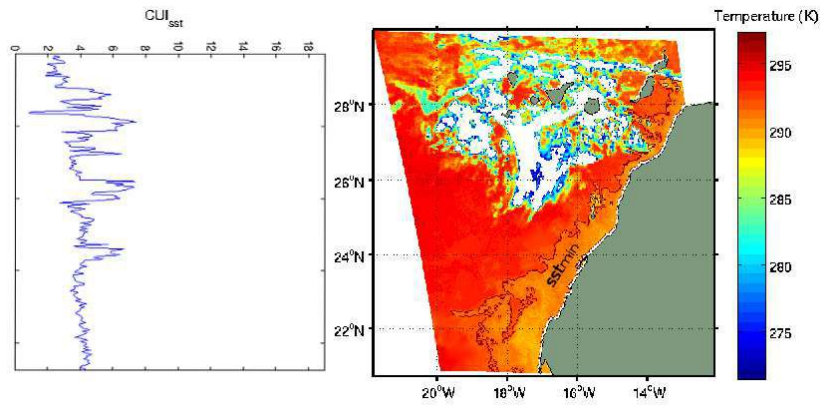

Fig. 5: Right) Upwelling area was contoured, where all isolated pixel were eliminated. Left) Spatial coastal upwelling index using the proposed method.

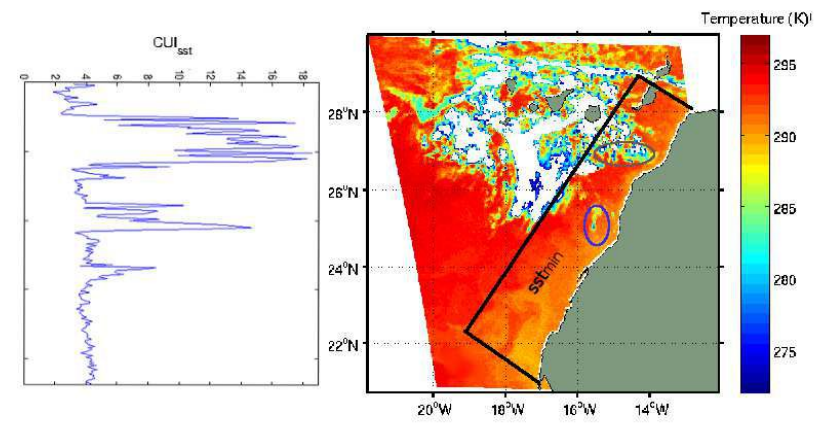

Fig. 6: Right)An example of the inshore of the classical way. Left) Spatial coastal upwelling index using the proposed classical way.

upwelling intensity shows the lowest values along the coast.

\section{CONCLUSION}

This paper deals with the developement of a new methodology to improve the SST-based CUI, by considering the nature of the upwelling areas and its dynamical spatial distribution. The improvement over simplest SST-based indices was in the extraction procedure of the minimal SST reference, which start by the delimitation of the upwelling area which performed under two step, the first one was to identify the upwelling area as an homogeneous set of pixel, while the second is to extract and filter out the noisy structures in the offshore waters not belonging to the upwelling area by using region-growing process. In summary, the proposed methodology has demonstrated an excellent performance and rigorous calculation of the coastal upwelling index.

\section{ACKNOWLEDGEMENT}

This work is funded by the French-Morrocan research program Toubkal/16/ 24 and PPR2-Minaoui.

\section{REFERENCES}

[1] Isabel Ambar and Joaquim Dias. Remote sensing of coastal upwelling in the north-eastern atlantic ocean. In Remote Sensing of the European Seas, pages 141-152. Springer, 2008.

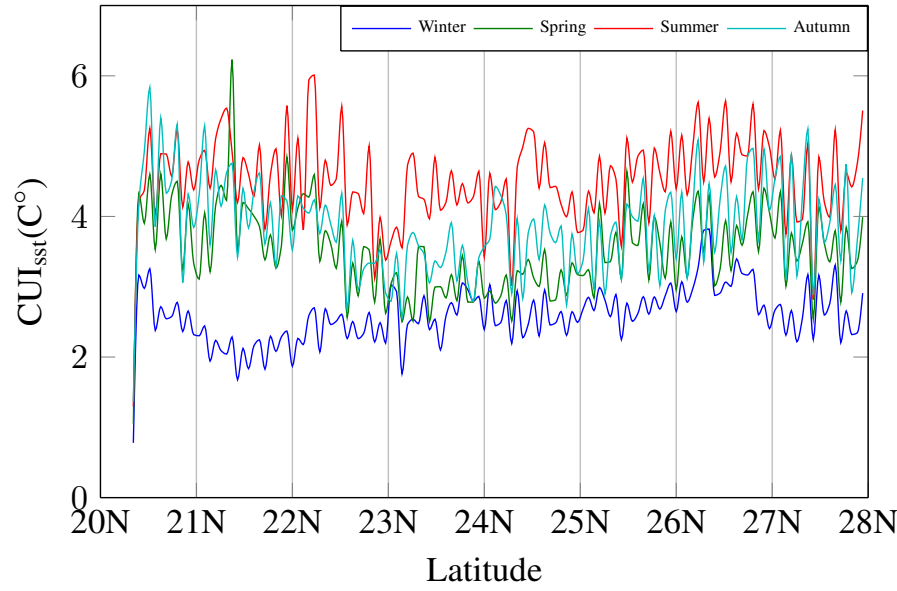

Fig. 7: Space-seasonal variability of the $C U T_{s s t}$, averaged over the 86 SST images.

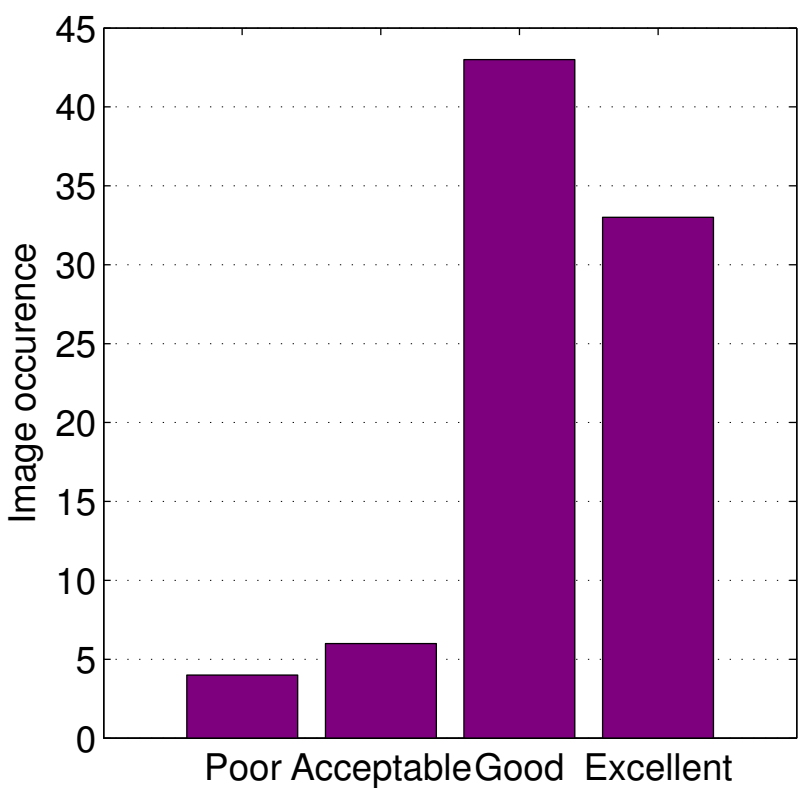

Fig. 8: Qualitative evaluation made by the oceanographer of the 86 SST images for the proposed methodology

[2] Aïssa Benazzouz, Soumia Mordane, Abdellatif Orbi, Mohamed Chagdali, Karim Hilmi, Abderrahman Atillah, Josep Lluís Pelegrí, and Demarcq Hervé. An improved coastal upwelling index from sea surface temperature using satellite-based approach-the case of the canary current upwelling system. Continental Shelf Research, 81:38-54, 2014.

[3] Yian-Leng Chang and Xiaobo Li. Adaptive image region-growing. Image Processing, IEEE Transactions on, 3(6):868-872, 1994.

[4] Swapnil Chaudhari, Ramprasad Balasubramanian, and Avijit Gangopadhyay. Upwelling detection in avhrr sea surface temperature (sst) images using neural-network framework. In Geoscience and Remote Sensing Symposium, 2008. IGARSS 2008. IEEE International, volume 4, pages IV-926. IEEE, 2008.

[5] Hervé DEMARCQ and Valérie FAURE. Coastal upwelling and associated retention indices derived from satellite sst. application to octopus vulgaris recruitment. Oceanologica Acta, 23(4):391-408, 2000.

[6] V Walfrid Ekman. On the influence of the earth \'s rotation on ocean currents. Ark. Mat. Astron. Fys., 2:1-53, 1905.

[7] Michael H Freilich and R Scott Dunbar. The accuracy of the nscat 1 vec- 
tor winds: Comparisons with national data buoy center buoys. Journal of Geophysical Research: Oceans (1978-2012), 104(C5):11231-11246, 1999.

[8] Annalisa Griffa, AD Kirwan Jr, Arthur J Mariano, Tamay Özgökmen, and $\mathrm{H}$ Thomas Rossby. Lagrangian analysis and prediction of coastal and ocean dynamics. Cambridge University Press, 2007.

[9] Anil K Jain and Richard C Dubes. Algorithms for clustering data. Prentice-Hall, Inc., 1988.

[10] Cyril Lathuilière, Vincent Echevin, and Marina Lévy. Seasonal and intraseasonal surface chlorophyll-a variability along the northwest african coast. Journal of Geophysical Research: Oceans, 113(C5), 2008.

[11] Carl G Looney. A fuzzy clustering and fuzzy merging algorithm. Reno, NV, 1999.

[12] Leo Nykjær and Lieve Van Camp. Seasonal and interannual variability of coastal upwelling along northwest africa and portugal from 1981 to 1991. Journal of Geophysical Research: Oceans, 99(C7):14197-14207, 1994.

[13] Theo Pavlidis and Yuh-Tay Liow. Integrating region growing and edge detection. Pattern Analysis and Machine Intelligence, IEEE Transactions on, 12(3):225-233, 1990.

[14] John A Richards. Clustering and unsupervised classification. In Remote Sensing Digital Image Analysis, pages 319-341. Springer, 2013.

[15] A Miguel P Santos, Alexander S Kazmin, and Alvaro Peliz. Decadal changes in the canary upwelling system as revealed by satellite observations: their impact on productivity. Journal of Marine Research, 63(2):359-379, 2005.

[16] Ayoub Tamim, Khalid Minaoui, Khalid Daoudi, Hussein Yahia, Abderrahman Atillah, and Driss Aboutajdine. An efficient tool for automatic delimitation of moroccan coastal upwelling using sst images. Geoscience and Remote Sensing Letters, IEEE, 12(4):875-879, 2015.

[17] L Van Camp, L Nykjaer, E Mittelstaedt, and P Schlittenhardt. Upwelling and boundary circulation off northwest africa as depicted by infrared and visible satellite observations. Progress in Oceanography, 26(4):357-402, 1991. 